Bull. Korean Math. Soc. 47 (2010), No. 6, pp. 1311-1327

DOI 10.4134/BKMS.2010.47.6.1311

\title{
GOTTLIEB SUBSETS WITH RESPECT TO A MORPHISM IN THE CATEGORY OF PAIRS
}

\author{
Jiyean Kim and Kee Young Lee
}

\begin{abstract}
We introduce the concept of cyclic morphisms with respect to a morphism in the category of pairs as a generalization of the concept of cyclic maps and we use the concept to obtain certain sets of homotopy classes in the category of pairs. For these sets, we get complete or partial answers to the following questions: (1) Is the concept the most general concept in the class of all concepts of generalized Gottlieb subsets introduced by many authors until now? (2) Are they homotopy invariants in the category of pairs? (3) When do they have a group structure?
\end{abstract}

\section{Introduction}

In his paper, "Generalized Gottlieb group" [13], K. Varadarajian introduced the concept of cyclic maps as a generalization of cyclic homotopies introduced by D. H. Gottlieb [2]. He also studied the concept of cocyclic maps as a dual concept of cyclic maps to fit well in the general set-up of Eckmann-Hilton duality. He studied the role of cyclic maps and cocyclic maps in the set up of Eckmann-Hilton duality. In [6, 8], Lee and Woo introduced the concept of the G-sequence under the homotopy sequence and its generalization. Moreover, they introduced the concept of cyclic morphisms with respect to a continuous map as a generalization of the concept of cyclic maps in [7].

It is our purpose in this paper to apply these concepts to objects in the category of pairs to induce further generalizations of cyclic maps appeared in the set up of Eckmann-Hilton duality and make further studies of the generalized Gottlieb subgroup. We introduce the concept of "cyclic morphism with respect to a morphism" in the category of pairs as a generalization of the cyclic map and we use the concept to define certain sets of homotopy classes in the category of pairs. We study the conditions for the sets to be homotopy invariants or groups. We also use the sets to study the role of cyclic morphisms in the category of pairs.

Received May 8, 2009.

2000 Mathematics Subject Classification. Primary 55Q05; Secondary 55P30.

Key words and phrases. category of pairs, cyclic map, cyclic morphism, generalized Gottlieb subset.

This work was supported by a Korea University Grant. 
In Section 2, we review the concepts of cyclic maps [13] and cyclic morphisms with respect to a continuous map in the category of pairs studied in [7].

In Section 3, we generalize the concept of cyclic morphisms with respect to a continuous map to that of cyclic morphisms with respect to a morphism and study the sets of homotopy classes of cyclic morphisms with respect to a morphism in the category of pairs.

In Section 4, we study properties of cyclic morphisms with respect to a morphism in the category of pairs. Especially, we characterize the cyclic morphisms with respect to a morphism by certain sets of homotopy classes in the category of pairs. Moreover, we find some conditions such that the sets are homotopy invariants in the category of pairs.

In Section 5, we find some sufficient conditions to ensure the set to be a group.

Throughout this paper, all spaces will be connected, based and of the homotopy type of CW-complexes. Hence the exponential law of function spaces holds and all base points denoted by $*$ are non-degenerate.

\section{Preliminaries}

In this paper, we shall consider two kinds of well-known categories in [4].

First, we shall consider the category of topological spaces with base points. The objects are pairs $(X, *)$ with $X$ a space and $*$ in $X$, and the maps are maps of these spaces which carry base point into base point. All homotopies considered will keep base points fixed. $\Pi(A, B)$ is the collection of homotopy classes of maps of $A$ into $B$. In the case $A=S^{n}$, the $n$-sphere, $\Pi(A, B)$ is a group, namely the $n$-th homotopy group. It is also well known that if $A$ has a coH-structure, $\Pi(A, B)$ has a group structure for all $B$ and if $B$ has a H-structure, $\Pi(A, B)$ has a group structure for all $A$. The reduced suspension of $X$, denoted $\Sigma X$, is the space obtained from $X \times I$ by identifying $X \times 0 \cup X \times 1 \cup * \times I$ to *, where $I$ is the unit interval $I=[0,1]$. More generally, we denote the $n$-folds suspension of the spaces $X$ by $\Sigma^{n} X \equiv \Sigma\left(\Sigma^{n-1} X\right)$. If $\alpha: X \rightarrow Y$ is a map, then the $n$-suspension map $\Sigma^{n} \alpha: \Sigma^{n} X \rightarrow \Sigma^{n} Y$ of $\alpha$ is the map defined by $\Sigma^{n} \alpha\left(\left\langle x, t_{1}, \ldots, t_{n}\right\rangle\right)=\left\langle\alpha(x), t_{1}, \ldots, t_{n}\right\rangle$, where $\left\langle x, t_{1}, \ldots, t_{n}\right\rangle$ is an element of $\Sigma^{n} X$ which is the equivalence class of $\left(x, t_{1}, \ldots, t_{n}\right) \in X \times I^{n}$. It is well-known fact that $S^{n}=\Sigma^{n}\left(S^{0}\right)$. The cone of $X$, denoted by $C X$, is the space obtained from $X \times I$ by pinching $X \times 1 \cup * \times I$ to $*$. Clearly $X$ is embedded in $C X$ by the natural inclusion map $x \rightarrow(x, 0)$. If we now squeeze $X \subset C X$ to $*$ we obtain the second definition of $\Sigma X$ which is obviously equivalent to the first. We can summarize by saying we have the following "exact" sequence of spaces:

$$
* \rightarrow X \rightarrow C X \rightarrow \Sigma X \rightarrow * \text {. }
$$

There is a natural map $\phi^{\prime}: \Sigma X \rightarrow \Sigma X \vee \Sigma X$ defined by

$$
\phi^{\prime}(x, t)= \begin{cases}((\langle x, 2 t\rangle, *) & \text { if } 0 \leq t \leq 1 / 2 \\ (*,\langle x, 2 t-1\rangle) & \text { if } 1 / 2 \leq t \leq 1\end{cases}
$$


It is easy to show that $\phi^{\prime}$ is a co-H-structure on $\Sigma X$. Thus for $n \geq 1$, the set $\Pi\left(\Sigma^{n} X, B\right) \equiv \Pi_{n}(X, B)$ is a group.

As the dual concept of the suspension of a space, we here recall the concept of the loop space. Let us denote the collection of mappings of $X$ into $Y$ with the compact-open topology by $Y^{X}$. The loop space of $X, \Omega X$, is the subspace of $X^{I}$ characterized by $\ell \in \Omega X$ such that $\ell(0)=\ell(1)=*$. There is a natural map

$$
\phi: \Omega X \times \Omega X \rightarrow \Omega X
$$

defined by

$$
\phi\left(\ell_{1}, \ell_{2}\right)(t)= \begin{cases}\ell_{1}(2 t) & \text { if } 0 \leq t \leq 1 / 2 \\ \ell_{2}(2 t-1) & \text { if } 1 / 2 \leq t \leq 1\end{cases}
$$

Continuity of $\phi$ follows from a standard argument in the compact open topology. It is easy to see that $\phi$ is a H-structure on $\Omega X$.

Given a map $f: \Sigma A \rightarrow B$, we define the adjoint map of $f \kappa(f)=\bar{f}: A \rightarrow$ $\Omega B$ by

$$
\bar{f}(a)(t)=f\langle a, t\rangle .
$$

A standard topological argument shows that $\bar{f}$ is continuous if and only if $f$ is continuous. Thus $\kappa$ is one to one correspondence between $B^{\Sigma A}$ and $(\Omega B)^{A}$.

Any map $g: A^{\prime} \rightarrow A$ induces a transformation $g^{*}: \Pi(A, B) \rightarrow \Pi\left(A^{\prime}, B\right)$ defined by $g^{*}([f])=[f \circ g]$, where the bracket '[ ]' denotes the homotopy class of the enclosed map. Clearly $(g h)^{*}=h^{*} g^{*}$. Similarly, $h: B \rightarrow B^{\prime}$ induces a transformation $h_{*}: \Pi(A, B) \rightarrow \Pi\left(A, B^{\prime}\right)$ defined by $h_{*}([f])=[h \circ f]$. The identity map on $X$ will be denoted by $1_{X}$ or $i d_{X}$, $*$ shall stand for the base point of a space and also the map which takes everything into the base point.

Second, we shall consider the category of pairs [4]. The category of pairs is the category in which the "object" are maps $(A, *) \rightarrow(B, *)$ and a "map" from $\alpha$ to $\beta$ is a pair of maps $\left(f_{1}, f_{2}\right)$ such that the diagram

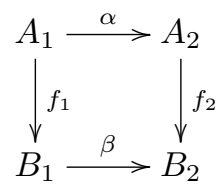

is commutative, i.e., $\beta f_{1}=f_{2} \alpha$. We shall call the maps in this category just "morphisms" to distinguish from maps between spaces. Moreover, we shall sometimes denote the morphism by the formula:

$$
\left(f_{1}, f_{2}\right): \alpha\left(A_{1} \rightarrow A_{2}\right) \rightarrow \beta\left(B_{1} \rightarrow B_{2}\right)
$$

or more simply,

$$
\left(f_{1}, f_{2}\right): \alpha \rightarrow \beta
$$

Two morphisms $\left(f_{1}, f_{2}\right),\left(g_{1}, g_{2}\right): \alpha \rightarrow \beta$ are called homotopic if there is a morphism $\left(H_{1}, H_{2}\right): \alpha \times 1_{I} \rightarrow \beta$ such that $H_{1}$ is a homotopy between $f_{1}$ and 
$g_{1}$ and $H_{2}$ is a homotopy between $f_{2}$ and $g_{2}$, where $1_{I}$ is the identity map of the unit interval $I$ into itself.

The set $\Pi(\alpha, \beta)$ is the family of all homotopy classes of morphisms from $\alpha$ to $\beta$ in the category of pairs. In particular, $\Pi_{n}(\alpha, \beta)=\Pi\left(\Sigma^{n} \alpha, \beta\right)$ is a group if $n \geq 1$ and abelian $n \geq 2$. If $\alpha=i_{n}: \Sigma^{n-1} A \rightarrow C \Sigma^{n-1} A$ is the natural inclusion, $\Pi(\alpha, \beta)$ is denoted by $\Pi_{n}(A, \beta)$. If $\beta$ is inclusion and $A=S^{0}$, we get the ordinary relative homotopy groups. Furthermore, if $\beta: * \rightarrow B$, $\Pi_{n}(A, \beta)=\Pi_{n}(A, B)$ and if $\beta: B \rightarrow *, \Pi_{n}(A, \beta)=\Pi_{n-1}(A, B)$.

A map $f: A \rightarrow X$ is said to be cyclic [13] if there exists a map $H: A \times X \rightarrow$ $X$ such that the diagram

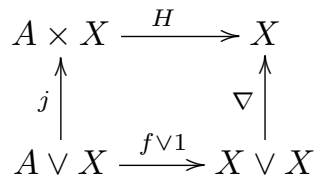

is homotopy commutative, where $j$ is the inclusion map and $\nabla$ is the folding map.

We denote the set of all homotopy classes of cyclic maps from $A$ to $X$ by $G(A, X)[13]$, that is, $G(A, X)=\{[f] \in \Pi(A, X) \mid f$ is a cyclic map $\}$, equivalently, $G(A, X)=\omega_{*}\left(\Pi\left(A, X^{X}\right)\right)$, where $\omega: X^{X} \rightarrow X$ is the evaluation map. In particular, $G\left(\Sigma^{n} A, X\right)$ is denoted by $G_{n}(A, X)$. Clearly, $\omega_{*}\left(\Pi_{n}\left(A, X^{X}\right)\right)=$ $G_{n}(A, X)$.

The subgroup $G_{n}(A, X)$ is a generalization of $G(A, X)$ and the Gottlieb group $G_{n}(X)[2]$. In fact $G_{0}(A, X)=G(A, X)$ and $G_{n}\left(S^{0}, X\right)=G_{n}(X)$.

Let $h: B \rightarrow X$ be a map. A map $f: A \rightarrow X$ is called a cyclic map with respect to $h: B \rightarrow X[10]$ if there exists a map $H: A \times B \rightarrow X$ such that the diagram

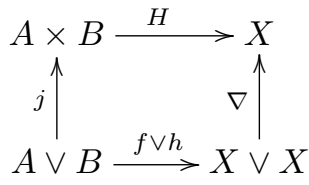

is homotopy commutative.

Here, we review the concept of cyclic morphisms with respect to a continuous map in the category of pairs studied in [7].

Let $h: X \rightarrow B_{1}$ be a map. A map $\left(f_{1}, f_{2}\right): \alpha \rightarrow \beta$ is called a cyclic morphism with respect to $h$ if there exists a map $\left(H_{1}, H_{2}\right): \alpha \times 1_{X} \rightarrow \beta$ such that $\left.\left(H_{1}, H_{2}\right)\right|_{\alpha}=\left(f_{1}, f_{2}\right)$ and $\left.\left(H_{1}, H_{2}\right)\right|_{1_{X}}=(h, \beta h)$, that is, the following 
diagram commutes:

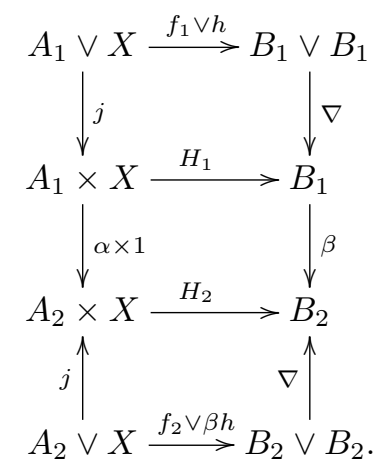

The morphism $\left(H_{1}, H_{2}\right)$ is called an affiliated morphism of $\left(f_{1}, f_{2}\right)$ with respect to $h$. If $h: B_{1} \rightarrow B_{1}$ is the identity, then $\left(f_{1}, f_{2}\right)$ is called just a cyclic morphism.

We define the subset $\mathcal{G}^{h}(\alpha, \beta)$ of $\Pi(\alpha, \beta)$ as the set of homotopy classes of cyclic morphisms with respect to $h$. That is,

$\mathcal{G}^{h}(\alpha, \beta)=\left\{\left[f_{1}, f_{2}\right] \in \Pi(\alpha, \beta) \mid\left(f_{1}, f_{2}\right)\right.$ is a cyclic morphism with respect to $\left.h\right\}$.

We denote $\mathcal{G}^{h}\left(\Sigma^{n} \alpha, \beta\right)$ by $\mathcal{G}_{n}^{h}(\alpha, \beta)$, where $\Sigma^{n} \alpha: \Sigma^{n} A_{1} \rightarrow \Sigma^{n} A_{2}$ is the suspension map of $\alpha$. In particular, if $i_{n}: \Sigma^{n} A \rightarrow C \Sigma^{n} A$ is the natural inclusion, then we denote $\mathcal{G}^{h}\left(i_{n}, \beta\right)$ by $\mathcal{G}_{n}^{h}(A, \beta)$. Moreover, we denote $\mathcal{G}_{n}^{h}(A, \beta)$ by $\mathcal{G}_{n}(A, \beta)$ if $h: B_{1} \rightarrow B_{1}$ is the identity map. $\mathcal{G}_{n}(A, \beta)$ is a generalization of $G_{n}^{\text {Rel }}\left(B_{2}, B_{1}\right)$ because $\mathcal{G}_{n}\left(S^{0}, i\right)=G_{n}^{\text {Rel }}\left(B_{2}, B_{1}\right)$, where $i: B_{1} \rightarrow B_{2}$ are the inclusion.

Define $\bar{\beta}:\left(B_{1}^{X}, h\right) \rightarrow\left(B_{2}^{X}, \beta h\right)$ by $\bar{\beta}(g)=\beta g$, where $\beta: B_{1} \rightarrow B_{2}$ is a map and let $\omega_{1}: B_{1}^{X} \rightarrow B_{1}$ and $\omega_{2}: B_{2}^{X} \rightarrow B_{2}$ be evaluation maps. Then $\left(\omega_{1}, \omega_{2}\right)$ : $\bar{\beta} \rightarrow \beta$ is a morphism and it induces a map $\left(\omega_{1}, \omega_{2}\right)_{*}: \Pi(\alpha, \bar{\beta}) \rightarrow \Pi(\alpha, \beta)$.

Theorem $2.1([7])$. Let $\beta: B_{1} \rightarrow B_{2}$ be a map and $\bar{\beta}:\left(B_{1}^{X}, h\right) \rightarrow\left(B_{2}^{X}, \beta h\right)$ be the map mentioned above. Then $\left(\omega_{1}, \omega_{2}\right)_{*} \Pi(\alpha, \bar{\beta})=\mathcal{G}^{h}(\alpha, \beta)$.

By above theorem, if $\alpha$ is a suspension map, then $\mathcal{G}^{h}(\alpha, \beta)$ is a group. In particular, $\mathcal{G}_{n}^{h}(\alpha, \beta)$ is a group, for $n \geq 1$.

Let $\left(g_{1}, g_{2}\right): \gamma \rightarrow \alpha$ be a morphism. If $\left(f_{1}, f_{2}\right): \alpha \rightarrow \beta$ is a cyclic morphism with respect to $h$, then the composition $\left(f_{1}, f_{2}\right) \circ\left(g_{1}, g_{2}\right): \gamma \rightarrow \beta$ is a cyclic morphism with respect to $h$. Thus the induced map $\left(g_{1}, g_{2}\right)^{*}: \Pi(\alpha, \beta) \rightarrow \Pi(\gamma, \beta)$ carries $\mathcal{G}^{h}(\alpha, \beta)$ into $\mathcal{G}^{h}(\gamma, \beta)$. Moreover, if $\left(g_{1}, g_{2}\right): \alpha \rightarrow \gamma$, then the induced map $\left(g_{1}, g_{2}\right)_{*}: \Pi(\alpha, \beta) \rightarrow \Pi(\alpha, \gamma)$ carries $\mathcal{G}^{h}(\alpha, \beta)$ into $\mathcal{G}^{g_{1} h}(\alpha, \gamma)$. If $\alpha \times 1_{X}: A_{1} \times X \rightarrow A_{2} \times X$ is a cofibration, then $\mathcal{G}^{h}(\alpha, \beta)$ is determined by the homotopy class of $h$. That is, if $h$ is homotopic to $h^{\prime}$, then $\mathcal{G}^{h}(\alpha, \beta)=\mathcal{G}^{h^{\prime}}(\alpha, \beta)$. Suppose $\alpha$ is a cofibration. Then if $\left(g_{1}, g_{2}\right): \gamma \rightarrow \alpha$ is a homotopy equivalence, $\left(g_{1}, g_{2}\right)^{*}: \mathcal{G}^{h}(\alpha, \beta) \rightarrow \mathcal{G}^{h}(\gamma, \beta)$ is an isomorphism. Similarly, if $\left(g_{1}, g_{2}\right): \alpha \rightarrow \gamma$ is a homotopy equivalence, then $\left(g_{1}, g_{2}\right)_{*}: \mathcal{G}^{h}(\alpha, \beta) \rightarrow \mathcal{G}^{g_{1} h}(\alpha, \gamma)$ is an isomorphism. Therefore we have the following theorem. 
Theorem 2.2 ([7]). The subgroup $\mathcal{G}_{n}(A, \beta)$ of $\Pi_{n}(A, \beta)$ is a homotopy invariant with respect to two variables.

\section{Cyclic morphisms with respect to a morphism and their homotopy classes}

In this section, we introduce the concept of cyclic morphisms with respect to a morphism and study the set of their homotopy classes in the category of pairs.

Definition. Let $\alpha: A_{1} \rightarrow A_{2}, \beta: B_{1} \rightarrow B_{2}$ and $\eta: X \rightarrow Y$ be objects and $\left(h_{1}, h_{2}\right): \eta \rightarrow \beta$ be a morphism in the category of pairs. A map $\left(f_{1}, f_{2}\right)$ : $\alpha \rightarrow \beta$ is called a cyclic morphism with respect to $\left(h_{1}, h_{2}\right)$ if there exists a map $\left(H_{1}, H_{2}\right): \alpha \times \eta \rightarrow \beta$ such that $\left.\left(H_{1}, H_{2}\right)\right|_{\alpha}=\left(f_{1}, f_{2}\right)$ and $\left.\left(H_{1}, H_{2}\right)\right|_{\eta}=\left(h_{1}, h_{2}\right)$, that is, the following diagram commutes:

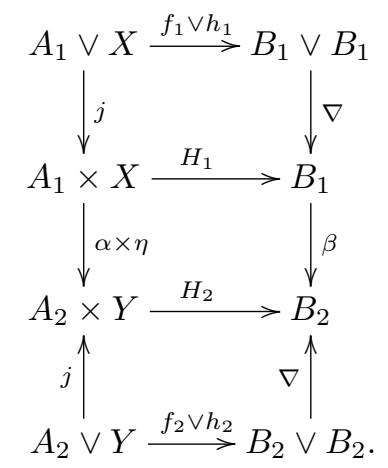

In this case, the morphism $\left(H_{1}, H_{2}\right)$ is called $\left(h_{1}, h_{2}\right)$-affiliated morphism of $\left(f_{1}, f_{2}\right)$ or $\left(f_{1}, f_{2}\right)$ has $\left(h_{1}, h_{2}\right)$-affiliated morphism $\left(H_{1}, H_{2}\right)$.

Remark 1. If $\eta=i d_{X}$, then it is clear that $\left(f_{1}, f_{2}\right)$ is a cyclic morphism with respect to $\left(h_{1}, h_{2}\right)$ if and only if $\left(f_{1}, f_{2}\right)$ is a cyclic morphism with respect to $h_{1}$. If $B_{2}=*$, then it is clear that $\left(f_{1}, f_{2}\right)$ is a cyclic morphism with respect to $\left(h_{1}, h_{2}\right)$ if and only if $f_{1}$ is a cyclic map with respect to $h_{1}$. In general, if $\left(f_{1}, f_{2}\right)$ is a cyclic morphism with respect to $\left(h_{1}, h_{2}\right)$, then $f_{1}$ is cyclic with respect to $h_{1}$ and $f_{2}$ is cyclic with respect to $h_{2}$. Moreover, if $i_{n}: S^{n-1} \rightarrow B^{n}$ and $i_{A}: A \rightarrow X$ are the inclusions, then it can be shown easily that a pair map $f:\left(B^{n}, S^{n-1}\right) \rightarrow(X, A)$ is relative cyclic if and only if $\left(\left.f\right|_{S^{n-1}}, f\right): i_{n} \rightarrow i_{A}$ is cyclic morphism [7]. So the concept of the cyclic morphism with respect to a morphism is a generalization of the cyclic morphism with respect to a map and the relative cyclic map.

Definition. We define the subset $\mathcal{G}^{\left(h_{1}, h_{2}\right)}(\alpha, \beta)$ of $\Pi(\alpha, \beta)$ as the set of homotopy classes of all cyclic morphisms with respect to $\left(h_{1}, h_{2}\right): \eta \rightarrow \beta$. That is,

$\mathcal{G}^{\left(h_{1}, h_{2}\right)}(\alpha, \beta)=\left\{\left[f_{1}, f_{2}\right] \in \Pi(\alpha, \beta) \mid\left(f_{1}, f_{2}\right)\right.$ : cyclic morphism w.r.t $\left.\left(h_{1}, h_{2}\right)\right\}$. 
$\mathcal{G}^{\left(h_{1}, h_{2}\right)}(\alpha, \beta)$ is called Gottlieb subset of $\Pi(\alpha, \beta)$ with respect to $\left(h_{1}, h_{2}\right)$ in the category of pairs.

For this set, we have three kinds of questions:

(1) Is it the most general concept in the class of all concepts of generalized Gottlieb subsets introduced by many authors until now?

(2) Is it homotopy invariant in the category of pairs?

(3) When does it have a group structure?

In this paper, we shall give answers to these questions.

By above definition, if $\left[g_{1}, g_{2}\right] \in \mathcal{G}^{\left(h_{1}, h_{2}\right)}(\alpha, \beta)$, then there exists a morphism $\left(f_{1}, f_{2}\right): \alpha \rightarrow \beta$ which is a cyclic morphism with respect to $\left(h_{1}, h_{2}\right)$ and homotopic to $\left(g_{1}, g_{2}\right)$ in the category of pairs. Thus there is not a problem in the definition of Gottlieb subset with respect to a morphism in the category of pairs. But we are interested in the statement that the homotopic relation preserves the cyclicity with respect to a morphism in the category of pairs, that is, if two morphisms $\left(f_{1}, f_{2}\right)$ and $\left(g_{1}, g_{2}\right)$ from $\alpha$ to $\beta$ are homotopic and $\left(f_{1}, f_{2}\right)$ is cyclic with respect to a morphism $\left(h_{1}, h_{2}\right)$, then $\left(g_{1}, g_{2}\right)$ is cyclic with respect to $\left(h_{1}, h_{2}\right)$. Is it true or false? If it is not true in general, when is it true? In the following lemma, we shall concentrate on solving this problem.

Lemma 3.1. Let $\eta: X \rightarrow Y$ be a homeomorphism and $\left(h_{1}, h_{2}\right): \eta \rightarrow \beta$ be a morphism for a map $\beta: B_{1} \rightarrow B_{2}$. If $\alpha: A_{1} \rightarrow A_{2}$ is an inclusion, two morphisms $\left(f_{1}, f_{2}\right)$ and $\left(g_{1}, g_{2}\right)$ from $\alpha$ to $\beta$ are homotopic and $\left(f_{1}, f_{2}\right)$ is cyclic with respect to a morphism $\left(h_{1}, h_{2}\right)$, then $\left(g_{1}, g_{2}\right)$ is cyclic with respect to $\left(h_{1}, h_{2}\right)$.

Proof. Suppose $\left(f_{1}, f_{2}\right)$ and $\left(g_{1}, g_{2}\right)$ are homotopic and $\left(f_{1}, f_{2}\right)$ is cyclic with respect to $\left(h_{1}, h_{2}\right)$. Then there is a homotopy $\left(F_{1}, F_{2}\right): \alpha \times i d_{I} \rightarrow \beta$ between $\left(f_{1}, f_{2}\right)$ and $\left(g_{1}, g_{2}\right)$, where $i d_{I}$ is the identity map on unit interval $I$. Moreover, there is an $\left(h_{1}, h_{2}\right)$-affiliated map $\left(H_{1}, H_{2}\right)$ from $\alpha \times \eta$ to $\beta$ of $\left(f_{1}, f_{2}\right)$. That is, we have commutative diagrams

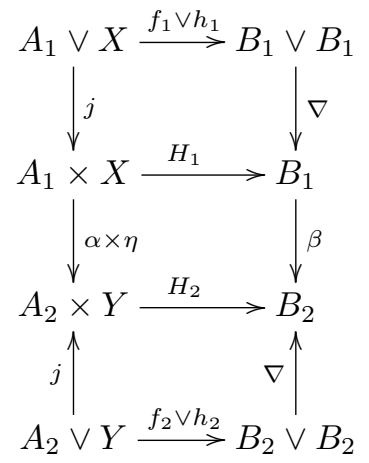


and

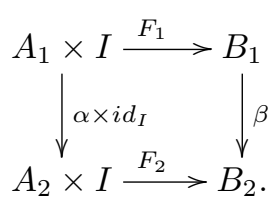

Define $\widehat{H}_{1}: A_{1} \times * \times I \sqcup * \times X \times I \sqcup A_{1} \times X \times 0 \rightarrow B_{1}$ by

$$
\widehat{H}_{1}(a, x, t)= \begin{cases}F_{1}(a, t) & \text { for }(a, x, t) \in A_{1} \times * \times I, \\ h_{1}(x) & \text { for }(a, x, t) \in * \times X \times I, \\ H_{1}(a, x) & \text { for }(a, x, t) \in A_{1} \times X \times 0 .\end{cases}
$$

Then $\widehat{H}_{1}$ is well-defined since $F_{1}(*, t)=*=h_{1}(*), F_{1}(a, 0)=f_{1}(a)=H_{1}(a, *)$ and $H_{1}(*, x)=h_{1}(x)$. But since $A_{1} \times * \cup * \times X$ is a sub-complex of $A_{1} \times X$, there is an extension $\bar{H}_{1}: A_{1} \times X \times I \rightarrow B_{1}$ of $\widehat{H}_{1}$. Similarly, we obtain another extension.

Define $\widehat{H}_{2}: A_{2} \times * \times I \sqcup A_{1} \times Y \times I \sqcup A_{2} \times Y \times 0 \rightarrow B_{2}$ by

$$
\widehat{H}_{2}(a, y, t)= \begin{cases}F_{2}(a, t) & \text { for }(a, y, t) \in A_{2} \times * \times I, \\ \beta \bar{H}_{1}\left(a, \eta^{-1}(y), t\right) & \text { for }(a, y, t) \in A_{1} \times Y \times I, \\ H_{2}(a, y) & \text { for }(a, y, t) \in A_{2} \times Y \times 0,\end{cases}
$$

where $\eta^{-1}: Y \rightarrow X$ is the inverse of $\eta$. Since $\beta \bar{H}_{1}\left(a, \eta^{-1}(*), t\right)=F_{2}(a, t)$ for $a \in A_{1}, \beta \bar{H}_{1}\left(a, \eta^{-1}(y), 0\right)=H_{2}(a, y)$ and $H_{2}(a, *)=f_{2}(a)=F_{2}(a, 0), \widehat{H}_{2}$ is well-defined. Since $A_{1} \times Y \cup A_{2} \times *$ is a sub-complex of $A_{2} \times Y$, there is an extension $\bar{H}_{2}: A_{2} \times Y \times I \rightarrow B_{2}$ of $\widehat{H}_{2}$. Let

$$
G_{1}=\left.\bar{H}_{1}\right|_{A_{1} \times X \times 1} \text { and } G_{2}=\left.\bar{H}_{2}\right|_{A_{2} \times Y \times 1} .
$$

Since for $(a, x) \in A_{1} \times X$,

$$
\begin{aligned}
G_{2}(\alpha \times \eta)(a, x) & =G_{2}(a, \eta(x))=\bar{H}_{2}(a, \eta(x), 1) \\
& =\widehat{H}_{2}(a, \eta(x), 1)=\beta \bar{H}_{1}\left(a, \eta^{-1}(\eta(x)), 1\right) \\
& =\beta \bar{H}_{1}(a, x, 1)=\beta G_{1}(a, x),
\end{aligned}
$$

$\left(G_{1}, G_{2}\right)$ is a morphism from $\alpha \times \eta$ to $\beta$. Moreover,

$$
\begin{gathered}
G_{1}(a, *)=\bar{H}_{1}(a, *, 1)=\widehat{H}_{1}(a, *, 1)=F_{1}(a, 1)=g_{1}(a), \\
G_{2}(a, *)=\bar{H}_{2}(a, *, 1)=\widehat{H}_{2}(a, *, 1)=F_{2}(a, 1)=g_{2}(a), \\
G_{1}(*, x)=\bar{H}_{1}(*, x, 1)=\widehat{H}_{1}(*, x, 1)=h_{1}(x),
\end{gathered}
$$

and

$$
\begin{aligned}
G_{2}(*, y) & =\bar{H}_{2}(*, y, 1)=\widehat{H}_{2}(*, y, 1)=\beta \widehat{H}_{1}\left(*, \eta^{-1}(y), 1\right) \\
& =\beta h_{1}\left(\eta^{-1}(y)\right)=h_{2}\left(\eta\left(\eta^{-1}(y)\right)\right)=h_{2}(y) .
\end{aligned}
$$

Therefore, $\left(G_{1}, G_{2}\right)$ is an $\left(h_{1}, h_{2}\right)$-affiliated morphism of $\left(g_{1}, g_{2}\right)$. 
By Remark 1 , the subset $\mathcal{G}^{\left(h_{1}, \beta h_{1}\right)}(\alpha, \beta)$ is just the subset $\mathcal{G}^{h_{1}}(\alpha, \beta)$. Moreover, $\mathcal{G}^{\left(h_{1}, *\right)}(\alpha, *)=G^{h_{1}}\left(A_{1}, B_{1}\right)$, where $\alpha: A_{1} \rightarrow B_{1}$ is a map. Here, we denote $\mathcal{G}^{\left(h_{1}, h_{2}\right)}\left(\Sigma^{n} \alpha, \beta\right)$ by $\mathcal{G}_{n}^{\left(h_{1}, h_{2}\right)}(\alpha, \beta)$, where $\Sigma^{n} \alpha: \Sigma^{n} A_{1} \rightarrow \Sigma^{n} A_{2}$ is the $n$-fold suspension map of $\alpha$. In particular, if $i_{n}: \Sigma^{n} A \rightarrow C \Sigma^{n} A$ is the natural inclusion, then we denote $\mathcal{G}^{\left(h_{1}, h_{2}\right)}\left(i_{n}, \beta\right)$ by $\mathcal{G}_{n}^{\left(h_{1}, h_{2}\right)}(A, \beta)$. Thus, we conclude that if $\eta=i d_{X}$ and $h_{2}: X \rightarrow *$, then $\mathcal{G}_{n}^{\left(h_{1}, h_{2}\right)}(A, \beta)$ is just $\mathcal{G}_{n}^{h_{1}}(A, \beta)$. Therefore, the Gottlieb subset of $\Pi(\alpha, \beta)$ with respect to a morphism $\left(h_{1}, h_{2}\right)$ in the category of pairs is the most general concept in the class of all generalized Gottlieb subsets introduced by many authors until now.

\section{Properties of cyclic morphisms with respect to a morphism}

Let $\alpha: A_{1} \rightarrow A_{2}, \beta: B_{1} \rightarrow B_{2}$ and $\gamma: C_{1} \rightarrow C_{2}$ be maps. Then we have the following lemma.

Lemma 4.1. Let $\eta: X \rightarrow Y$ be a map and $\left(h_{1}, h_{2}\right): \eta \rightarrow \beta$ and $\left(g_{1}, g_{2}\right)$ : $\gamma \rightarrow \alpha$ be morphisms. If $\left(f_{1}, f_{2}\right): \alpha \rightarrow \beta$ is a cyclic morphism with respect to $\left(h_{1}, h_{2}\right): \eta \rightarrow \beta$, then $\left(f_{1}, f_{2}\right) \circ\left(g_{1}, g_{2}\right): \gamma \rightarrow \beta$ is a cyclic morphism with respect to $\left(h_{1}, h_{2}\right)$.

Proof. Let $\left(H_{1}, H_{2}\right): \alpha \times \eta \rightarrow \beta$ be an $\left(h_{1}, h_{2}\right)$-affiliated morphism of $\left(f_{1}, f_{2}\right)$. Then $\left(H_{1}\left(g_{1} \times 1_{X}\right), H_{2}\left(g_{2} \times 1_{X}\right)\right): \gamma \times \eta \rightarrow \beta$ is an $\left(h_{1}, h_{2}\right)$-affiliated morphism of $\left(f_{1}, f_{2}\right) \circ\left(g_{1}, g_{2}\right)$. It is clear by the following commutative diagram;

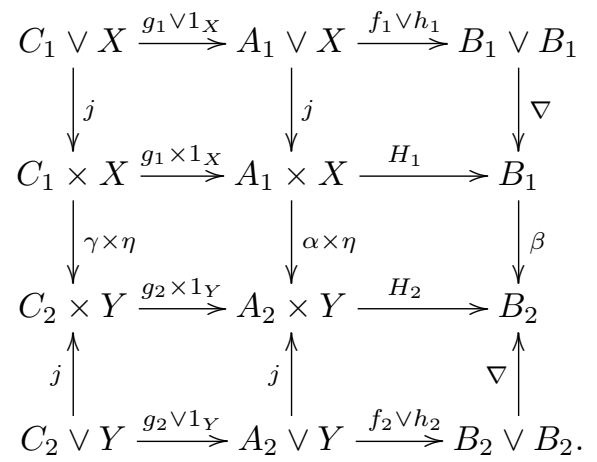

Corollary 1. If $\left(g_{1}, g_{2}\right): \gamma \rightarrow \alpha$ is a morphism, then the induced map $\left(g_{1}, g_{2}\right)^{*}: \Pi(\alpha, \beta) \rightarrow \Pi(\gamma, \beta)$ carries $\mathcal{G}^{\left(h_{1}, h_{2}\right)}(\alpha, \beta)$ into $\mathcal{G}^{\left(h_{1}, h_{2}\right)}(\gamma, \beta)$.

Corollary 1 shows that for fixed $\beta, \mathcal{G}^{\left(h_{1}, h_{2}\right)}(\alpha, \beta)$ is a homotopy invariant in the category of pairs. That is, we have the following theorem.

Theorem 4.2. Let $\left(g_{1}, g_{2}\right): \gamma \rightarrow \alpha$ be a homotopy equivalence. Then $\left(g_{1}, g_{2}\right)^{*}$ : $\mathcal{G}^{\left(h_{1}, h_{2}\right)}(\alpha, \beta) \rightarrow \mathcal{G}^{\left(h_{1}, h_{2}\right)}(\gamma, \beta)$ is an one-to-one correspondence. Especially, $\left(g_{1}, g_{2}\right)^{*}$ is an isomorphism provided that $\mathcal{G}^{\left(h_{1}, h_{2}\right)}(\alpha, \beta)$ and $\mathcal{G}^{\left(h_{1}, h_{2}\right)}(\gamma, \beta)$ are groups. 
Lemma 4.3. If $\left(g_{1}, g_{2}\right): \beta \rightarrow \gamma$ is a morphism and $\left(f_{1}, f_{2}\right): \alpha \rightarrow \beta$ is a cyclic morphism with respect to $\left(h_{1}, h_{2}\right): \eta \rightarrow \beta$, then the composition $\left(g_{1}, g_{2}\right) \circ\left(f_{1}, f_{2}\right)$ is a cyclic morphism with respect to $\left(g_{1} h_{1}, g_{2} h_{2}\right): \eta \rightarrow \gamma$.

Proof. Let $\left(H_{1}, H_{2}\right): \alpha \times \eta \rightarrow \beta$ be an $\left(h_{1}, h_{2}\right)$-affiliated morphism of $\left(f_{1}, f_{2}\right)$. Then $\left(g_{1} H_{1}, g_{2} H_{2}\right): \alpha \times \eta \rightarrow \gamma$ is an $\left(g_{1} h_{1}, g_{2} h_{2}\right)$-affiliated morphism of $\left(g_{1} f_{1}, g_{2} f_{2}\right)$.

Corollary 2. If $\left(g_{1}, g_{2}\right): \beta \rightarrow \gamma$ is a morphism, then the induced map $\left(g_{1}, g_{2}\right)_{*}: \Pi(\alpha, \beta) \rightarrow \Pi(\alpha, \gamma)$ carries $\mathcal{G}^{\left(h_{1}, h_{2}\right)}(\alpha, \beta)$ into $\mathcal{G}^{\left(g_{1} h_{1}, g_{2} h_{2}\right)}(\alpha, \gamma)$.

Is $\mathcal{G}^{\left(h_{1}, h_{2}\right)}(\alpha, \beta)$ a homotopy invariant in the category of pairs for fixed $\alpha$ ? It may not be true, since it does not follow from Lemma 4.3 or Corollary 2 as Theorem 4.2. But we can give a partial solution of this question, by proving the following lemma.

Lemma 4.4. Suppose $\alpha: A_{1} \rightarrow A_{2}$ is an inclusion and $\eta: X \rightarrow Y$ is a homeomorphism. Then if two morphisms $\left(h_{1}, h_{2}\right)$ and $\left(h_{1}^{\prime}, h_{2}^{\prime}\right)$ from $\eta$ to a map $\beta: B_{1} \rightarrow B_{2}$ are homotopic, $\mathcal{G}^{\left(h_{1}, h_{2}\right)}(\alpha, \beta)=\mathcal{G}^{\left(h_{1}^{\prime}, h_{2}^{\prime}\right)}(\alpha, \beta)$.

Proof. It is sufficient to show that one of them contains the other. Let us show that $\mathcal{G}^{\left(h_{1}, h_{2}\right)}(\alpha, \beta) \subset \mathcal{G}^{\left(h_{1}^{\prime}, h_{2}^{\prime}\right)}(\alpha, \beta)$. By Lemma 3.1, it is sufficient to show that there is an $\left(h_{1}^{\prime}, h_{2}^{\prime}\right)$-affiliated morphism of $\left(f_{1}, f_{2}\right)$ for each $\left[f_{1}, f_{2}\right] \in$ $\mathcal{G}^{\left(h_{1}, h_{2}\right)}(\alpha, \beta)$. Suppose $\left[f_{1}, f_{2}\right] \in \mathcal{G}^{\left(h_{1}, h_{2}\right)}(\alpha, \beta)$. Then there is an $\left(h_{1}, h_{2}\right)$ affiliated morphism of $\left(f_{1}, f_{2}\right)$, that is, there is a morphism $\left(H_{1}, H_{2}\right): \alpha \times \eta \rightarrow \beta$ such that $\left.H_{i}\right|_{A_{i} \times *}=f_{i}$ for $i=1,2,\left.H_{1}\right|_{* \times X}=h_{1}$ and $\left.H_{2}\right|_{* \times Y}=h_{2}$. Moreover, there is a homotopy $\left(F_{1}, F_{2}\right): \eta \times i d_{I} \rightarrow \beta$ between $\left(h_{1}, h_{2}\right)$ and $\left(h_{1}^{\prime}, h_{2}^{\prime}\right)$. Define $\widehat{H}_{1}: A_{1} \times * \times I \sqcup * \times X \times I \sqcup A_{1} \times X \times 0 \rightarrow B_{1}$ by

$$
\widehat{H}_{1}(a, x, t)= \begin{cases}f_{1}(a) & \text { for }(a, x, t) \in A_{1} \times * \times I, \\ F_{1}(x, t) & \text { for }(a, x, t) \in * \times X \times I, \\ H_{1}(a, x) & \text { for }(a, x, t) \in A_{1} \times X \times 0 .\end{cases}
$$

Then $\widehat{H}_{1}$ is well-defined. Since $A_{1} \times * \cup * \times X$ is a sub-complex of $A_{1} \times X$, there is an extension $\bar{H}_{1}: A_{1} \times X \times I \rightarrow B_{1}$ of $\widehat{H}_{1}$. Similarly, we obtain another extension.

$$
\begin{aligned}
& \text { Define } \widehat{H}_{2}: A_{2} \times * \times I \sqcup A_{1} \times Y \times I \sqcup A_{2} \times Y \times 0 \rightarrow B_{2} \text { by } \\
& \qquad \widehat{H}_{2}(a, y, t)= \begin{cases}f_{2}(a) & \text { for }(a, y, t) \in A_{2} \times * \times I, \\
\beta \bar{H}_{1}\left(a, \eta^{-1}(y), t\right) & \text { for }(a, y, t) \in A_{1} \times Y \times I, \\
H_{2}(a, y) & \text { for }(a, y, t) \in A_{2} \times Y \times 0,\end{cases}
\end{aligned}
$$

where $\eta^{-1}: Y \rightarrow X$ is the inverse of $\eta$. Since $\beta \bar{H}_{1}\left(a, \eta^{-1}(*), t\right)=\beta f_{1}(a)=$ $f_{2}(a)$ for $a \in A_{1}, \beta \bar{H}_{1}\left(a, \eta^{-1}(y), 0\right)=\beta H_{1}\left(a, \eta^{-1}(y)\right)=H_{2}(a, y)$ for $a \in A_{1}$ and $H_{2}(a, *)=f_{2}(a)$ for $a \in A_{2}, \widehat{H}_{2}$ is well-defined. Since $A_{1} \times Y \cup A_{2} \times *$ is a sub-complex of $A_{2} \times Y$, there is an extension $\bar{H}_{2}: A_{2} \times Y \times I \rightarrow B_{2}$ of $\widehat{H}_{2}$. Let

$$
G_{1}=\left.\bar{H}_{1}\right|_{A_{1} \times X \times 1} \text { and } G_{2}=\left.\bar{H}_{2}\right|_{A_{2} \times Y \times 1} \text {. }
$$


Since for $(a, x) \in A_{1} \times X$

$$
\begin{aligned}
G_{2}(\alpha \times \eta)(a, x) & =G_{2}(a, \eta(x))=\bar{H}_{2}(a, \eta(x), 1) \\
& =\widehat{H}_{2}(a, \eta(x), 1)=\beta \bar{H}_{1}\left(a, \eta^{-1}(\eta(x)), 1\right) \\
& =\beta \bar{H}_{1}(a, x, 1)=\beta G_{1}(a, x),
\end{aligned}
$$

$\left(G_{1}, G_{2}\right)$ is a morphism from $\alpha \times \eta$ to $\beta$. Moreover,

$$
\begin{gathered}
G_{1}(a, *)=\bar{H}_{1}(a, *, 1)=\widehat{H}_{1}(a, *, 1)=f_{1}(a) \text { for } a \in A_{1}, \\
G_{2}(a, *)=\bar{H}_{2}(a, *, 1)=\widehat{H}_{2}(a, *, 1)=f_{2}(a) \text { for } a \in A_{2}, \\
G_{1}(*, x)=\bar{H}_{1}(*, x, 1)=\widehat{H}_{1}(*, x, 1)=F(x, 1)=h_{1}^{\prime}(x) \text { for } x \in X,
\end{gathered}
$$

and for $y \in Y$

$$
\begin{aligned}
G_{2}(*, y) & =\bar{H}_{2}(*, y, 1)=\widehat{H}_{2}(*, y, 1)=\beta \bar{H}_{1}\left(*, \eta^{-1}(y), 1\right) \\
& =\beta F_{1}\left(\eta^{-1}(y), 1\right)=\beta h_{1}^{\prime}\left(\eta^{-1}(y), 1\right)=h_{2}^{\prime}\left(\eta\left(\eta^{-1}(y)\right)\right)=h_{2}^{\prime}(y) .
\end{aligned}
$$

Therefore, $\left(G_{1}, G_{2}\right)$ is an $\left(h_{1}^{\prime}, h_{2}^{\prime}\right)$-affiliated morphism of $\left(f_{1}, f_{2}\right)$.

Theorem 4.5. Suppose $\alpha: A_{1} \rightarrow A_{2}$ is an inclusion, $\eta: X \rightarrow Y$ is a homeomorphism and $\left(g_{1}, g_{2}\right): \beta \rightarrow \gamma$ is a homotopy equivalence. Then $\left(g_{1}, g_{2}\right)_{*}$ : $\mathcal{G}^{\left(h_{1}, h_{2}\right)}(\alpha, \beta) \rightarrow \mathcal{G}^{\left(g_{1} h_{1}, g_{2} h_{2}\right)}(\alpha, \gamma)$ is an one-to-one correspondence. Especially, $\left(g_{1}, g_{2}\right)_{*}$ is an isomorphism provided that $\mathcal{G}^{\left(h_{1}, h_{2}\right)}(\alpha, \beta)$ and $\mathcal{G}^{\left(g_{1} h_{1}, g_{2} h_{2}\right)}(\alpha, \gamma)$ are groups.

Proof. Let $\left(g_{1}^{\prime}, g_{2}^{\prime}\right): \gamma \rightarrow \beta$ be a homotopy inverse of $\left(g_{1}, g_{2}\right)$. Then

$$
\left(g_{1}^{\prime}, g_{2}^{\prime}\right)_{*}\left(\mathcal{G}^{\left(g_{1} h_{1}, g_{2} h_{2}\right)}(\alpha, \gamma)\right) \subset \mathcal{G}^{\left(g_{1}^{\prime} g_{1} h_{1}, g_{2}^{\prime} g_{2} h_{2}\right)}(\alpha, \beta)=\mathcal{G}^{\left(h_{1}, h_{2}\right)}(\alpha, \beta)
$$

by Corollary 2 and Lemma 4.4. Thus

$$
\left(g_{1}^{\prime}, g_{2}^{\prime}\right)_{*}: \mathcal{G}^{\left(g_{1} h_{1}, g_{2} h_{2}\right)}(\alpha, \gamma) \rightarrow \mathcal{G}^{\left(h_{1}, h_{2}\right)}(\alpha, \beta)
$$

is the inverse function of $\left(g_{1}, g_{2}\right)_{*}$.

Lemma 4.1 also gives us an information which characterizes the cyclic morphism as follows.

Theorem 4.6. Let $\beta: B_{1} \rightarrow B_{2}$ and $\eta: X \rightarrow Y$ be maps. If the identity morphism $\left(i d_{B_{1}}, i d_{B_{2}}\right): \beta \rightarrow \beta$ is cyclic with respect to a morphism $\left(h_{1}, h_{2}\right)$ : $\eta \rightarrow \beta$, then $\mathcal{G}^{\left(h_{1}, h_{2}\right)}(\alpha, \beta)=\Pi(\alpha, \beta)$ for any map $\alpha$.

Proof. Suppose $\left(i d_{B_{1}}, i d_{B_{2}}\right): \beta \rightarrow \beta$ is cyclic with respect to $\left(h_{1}, h_{2}\right)$ and $\left[f_{1}, f_{2}\right] \in \Pi(\alpha, \beta)$. Since $\left(f_{1}, f_{2}\right)=\left(i d_{B_{1}}, i d_{B_{2}}\right)\left(f_{1}, f_{2}\right),\left(f_{1}, f_{2}\right)$ is cyclic with respect to $\left(h_{1}, h_{2}\right)$ by Lemma 4.1 . Thus $\left[f_{1}, f_{2}\right] \in \mathcal{G}^{\left(h_{1}, h_{2}\right)}(\alpha, \beta)$.

The reverse implication may not be true because the homotopic relation does not preserve the cyclicity in the category of pairs in general. But under the hypothesis that $\beta: B_{1} \rightarrow B_{2}$ is an inclusion and $\eta: X \rightarrow Y$ is a homeomorphism, it is true and thus we have the following theorem. 
Theorem 4.7. Let $\beta: B_{1} \rightarrow B_{2}$ be an inclusion. Then the identity morphism $\left(i d_{B_{1}}, i d_{B_{2}}\right): \beta \rightarrow \beta$ is cyclic with respect to a morphism $\left(h_{1}, h_{2}\right): \eta \rightarrow \beta$ if and only if $\mathcal{G}^{\left(h_{1}, h_{2}\right)}(\alpha, \beta)=\Pi(\alpha, \beta)$ for each map $\alpha$.

Proof. By Theorem 4.6, it is sufficient to prove the 'only if' part. Suppose $\mathcal{G}^{\left(h_{1}, h_{2}\right)}(\alpha, \beta)=\Pi(\alpha, \beta)$ for each map $\alpha$. Especially, $\mathcal{G}^{\left(h_{1}, h_{2}\right)}(\beta, \beta)=\Pi(\beta, \beta)$. Thus

$$
\left[i d_{B_{1}}, i d_{B_{2}}\right] \in \Pi(\beta, \beta)=\mathcal{G}^{\left(h_{1}, h_{2}\right)}(\beta, \beta) .
$$

Therefore, there is a morphism $\left(f_{1}, f_{2}\right): \beta \rightarrow \beta$ which is cyclic with respect to $\left(h_{1}, h_{2}\right)$ and homotopic to $\left(i d_{B_{1}}, i d_{B_{2}}\right)$ in the category of pairs. By Lemma 3.1, $\left(i d_{B_{1}}, i d_{B_{2}}\right)$ is cyclic with respect to $\left(h_{1}, h_{2}\right)$.

\section{Group structures of Gottlieb subsets with respect to a morphism}

In this section, we shall investigate group structures of $\mathcal{G}^{\left(h_{1}, h_{2}\right)}(\alpha, \beta)$. First, we shall check the possibility that the Gottlieb subset with respect a morphism is an image of the induced map from an evaluation morphism in the category of pairs.

Let $\omega_{1}: B_{1}^{X} \rightarrow B_{1}$ and $\omega_{2}: B_{2}^{Y} \rightarrow B_{2}$ be evaluation maps at a base point $* \in X$ and $* \in Y$, respectively. Let $\bar{\beta}: B_{1}^{X} \rightarrow B_{2}^{Y}$ be an object and $\left(\omega_{1}, \omega_{2}\right): \bar{\beta} \rightarrow \beta$ a morphism. Then $\left(\omega_{1}, \omega_{2}\right)_{*}: \Pi(\alpha, \bar{\beta}) \rightarrow \Pi(\alpha, \beta)$ is a homomorphism.

Definition. An object $\eta: X \rightarrow Y$ is called a right invertible object if it has a right inverse, that is, there is an object $\theta: Y \rightarrow X$ such that $\eta \circ \theta=i d_{Y}$.

Example 1. (a) The identity map $i d_{X}: X \rightarrow X$ is a right invertible object.

(b) The natural projection $p: X \times Y \rightarrow Y$ is a right invertible object.

(c) Every homeomorphism is a right invertible object.

(d) Every Hurewicz fibration with a cross-section is a right invertible object.

Lemma 5.1. Let $\beta: B_{1} \rightarrow B_{2}$ and $\eta: X \rightarrow Y$ be objects and $\left(h_{1}, h_{2}\right): \eta \rightarrow \beta$ be a morphism. If $\eta$ is a right invertible object with the inverse $\theta$, then there exists an object $\bar{\beta}: B_{1}^{X} \rightarrow B_{2}^{Y}$ such that $\bar{\beta}\left(h_{1}\right)=h_{2}$, where $B_{1}^{X}$ and $B_{2}^{Y}$ are function spaces with compact open topology with base points $h_{1}, h_{2}$, respectively.

Proof. Define $\bar{\beta}: B_{1}^{X} \rightarrow B_{2}^{Y}$ by $\bar{\beta}(g)=\beta \circ g \circ \theta$ for each $g \in B_{1}^{X}$. Then $\bar{\beta}$ is continuous since the adjoint $\widehat{\bar{\beta}}$ of $\bar{\beta}$ is the composition $\widehat{\bar{\beta}}=\beta \circ$ ev $\circ\left(1_{B_{1}^{X}} \times \theta\right)$ and it is continuous, where $e v$ is the evaluation map from $B_{1}^{X} \times X$ to $B_{1}$ given by $\operatorname{ev}(f, x)=f(x)$. Moreover,

$$
\bar{\beta}\left(h_{1}\right)=\beta h_{1} \theta=h_{2} \eta \theta=h_{2} .
$$

Lemma 5.2. Let $\alpha: A_{1} \rightarrow A_{2}$ and $\beta: B_{1} \rightarrow B_{2}$ be objects, $\eta: X \rightarrow Y$ be a right invertible map with the inverse $\theta$ and $\left(h_{1}, h_{2}\right): \eta \rightarrow \beta$ be a morphism. Then $\mathcal{G}^{\left(h_{1}, h_{2}\right)}(\alpha, \beta)$ is contained in the image $\left(\omega_{1}, \omega_{2}\right)_{*} \Pi(\alpha, \bar{\beta})$. 
Proof. Let $\left[f_{1}, f_{2}\right] \in \mathcal{G}^{\left(h_{1}, h_{2}\right)}(\alpha, \beta)$. Then there exists a morphism $\left(H_{1}, H_{2}\right)$ : $\alpha \times \eta \rightarrow \beta$ such that the following diagram is commutative:

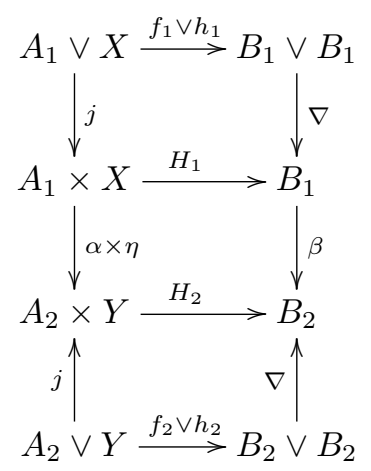

Consider the adjoint maps $\widetilde{H_{1}}$ and $\widetilde{H_{2}}$ of $H_{1}$ and $H_{2}$, respectively. Define $\bar{\beta}: B_{1}^{X} \rightarrow B_{2}^{Y}$ by $\bar{\beta}(g)=\beta \circ g \circ \theta$. Then $\left(\widetilde{H_{1}}, \widetilde{H_{2}}\right): \alpha \rightarrow \bar{\beta}$ is a morphism. In fact,

$$
\begin{aligned}
\bar{\beta} \widetilde{H_{1}}(a)(y) & =\left(\beta \circ \widetilde{H_{1}}(a) \circ \theta\right)(y)=\beta H_{1}(a, \theta(y))=H_{2}(\alpha(a), \eta \theta(y)) \\
& =\widetilde{H_{2}}(\alpha(a))(\eta \theta(y))=\widetilde{H_{2}}(\alpha(a))(y) .
\end{aligned}
$$

Moreover,

$$
\omega_{1} \widetilde{H_{1}}(a)=\widetilde{H_{1}}(a)(*)=H_{1}(a, *)=f_{1}(a), \omega_{2} \widetilde{H_{2}}(a)=\widetilde{H_{2}}(a)(*)=f_{2}(a) .
$$

Thus $\left[\widetilde{H_{1}}, \widetilde{H_{2}}\right] \in \Pi(\alpha, \bar{\beta})$ and $\left(\omega_{1}, \omega_{2}\right)_{*}\left[\widetilde{H_{1}}, \widetilde{H_{2}}\right]=\left[\omega_{1} \widetilde{H_{1}}, \omega_{2} \widetilde{H_{2}}\right]=\left[f_{1}, f_{2}\right]$. Therefore $\mathcal{G}^{\left(h_{1}, h_{2}\right)}(\alpha, \beta) \subset\left(\omega_{1}, \omega_{2}\right)_{*} \Pi(\alpha, \bar{\beta})$.

Lemma 5.3. Under the same hypothesis in Lemma 5.2, we have

$$
\left(\omega_{1}, \omega_{2}\right)_{*} \Pi(\alpha, \bar{\beta}) \subset \mathcal{G}^{\left(h_{1} \theta \eta, h_{2}\right)}(\alpha, \beta)
$$

for the object $\bar{\beta}: B_{1}^{X} \rightarrow B_{2}^{Y}$ given by $\bar{\beta}(g)=\beta g \theta$, where $\theta$ is a right inverse of $\eta$.

Proof. Let $\left[f_{1}, f_{2}\right] \in\left(\omega_{1}, \omega_{2}\right)_{*} \Pi(\alpha, \bar{\beta})$. Then there exists a morphism $\left(\bar{f}_{1}, \bar{f}_{2}\right)$ : $\alpha \rightarrow \bar{\beta}$ such that $\left(\omega_{1}, \omega_{2}\right)_{*}\left[\bar{f}_{1}, \bar{f}_{2}\right]=\left[f_{1}, f_{2}\right]$. By the definition of $\mathcal{G}^{\left(h_{1} \theta \eta, h_{2}\right)}(\alpha, \beta)$, we may assume $\left(\omega_{1}, \omega_{2}\right)_{*}\left(\bar{f}_{1}, \bar{f}_{2}\right)=\left(f_{1}, f_{2}\right)$. Consider the adjoint maps $\widetilde{\bar{f}}_{1}$ : $A_{1} \times X \rightarrow B_{1}$ and $\widetilde{\widetilde{f}}_{2}: A_{2} \times Y \rightarrow B_{2}$ of $\bar{f}_{1}$ and $\bar{f}_{2}$ respectively. Define $\widehat{f}_{1}=\widetilde{\bar{f}}_{1}\left(1_{A_{1}} \times \theta \eta\right)$ and $\widehat{f}_{2}=\widetilde{\bar{f}}_{2}$. Then $\left(\widehat{f}_{1}, \widehat{f}_{2}\right)$ is a morphism from $\alpha \times \eta$ to $\beta$. In fact,

$$
\begin{aligned}
\widehat{f}_{2}(\alpha \times \eta)(a, x) & =\widehat{f}_{2}(\alpha(a), \eta(x))=\bar{f}_{2} \alpha(x)(\eta(x)) \\
& =\overline{\beta f}_{1}(a)(\eta(x))=\beta \bar{f}_{1}(a) \theta \eta(x)=\beta \widetilde{\bar{f}}_{1}(a, \theta \eta(x)) \\
& =\beta \widetilde{\bar{f}}_{1}\left(1_{A_{1}} \times \theta \eta\right)(a, x)=\beta \widehat{f}_{1}(a, x) .
\end{aligned}
$$


Furthermore,

$$
\begin{gathered}
\widehat{f}_{1}(a, *)=\widetilde{\bar{f}}_{1}(a, \theta \eta(*))=\bar{f}_{1}(a)(*)=\omega_{1} \bar{f}_{1}(a)=f_{1}(a), \\
\widehat{f}_{1}(*, x)=\widetilde{\bar{f}}_{1}(*, \theta \eta(x))=\bar{f}_{1}(*) \theta \eta(x)=h_{1} \theta \eta(x), \\
\widehat{f}_{2}(a, *)=\bar{f}_{2}(a)(*)=\omega_{2} \bar{f}_{2}(a)=f_{2}(a)
\end{gathered}
$$

and

$$
\widehat{f}_{2}(*, x)=\bar{f}_{2}(*)(x)=h_{2} .
$$

Thus $\left[f_{1}, f_{2}\right] \in \mathcal{G}^{\left(h_{1} \theta \eta, h_{2}\right)}(\alpha, \beta)$.

By Lemma 5.2 and Lemma 5.3, we have the following theorem.

Theorem 5.4. Let $\alpha: A_{1} \rightarrow A_{2}$ and $\beta: B_{1} \rightarrow B_{2}$ be maps, $\eta: X \rightarrow Y$ be $a$ homeomorphism and $\left(h_{1}, h_{2}\right): \eta \rightarrow \beta$ be a morphism. Then we have

$$
\mathcal{G}^{\left(h_{1}, h_{2}\right)}(\alpha, \beta)=\left(\omega_{1}, \omega_{2}\right)_{*} \Pi(\alpha, \bar{\beta}),
$$

where $\bar{\beta}: B_{1}^{X} \rightarrow B_{2}^{Y}$ is defined by $\bar{\beta}(g)=\beta g \theta$ for $g \in B_{1}^{X}$ and the inverse $\theta$ of $\eta$.

Definition. A map $\alpha: A_{1} \rightarrow A_{2}$ is said to be co-H-object if there exists a morphism a morphism $\left(\phi_{1}, \phi_{2}\right): \alpha \rightarrow \alpha \vee \alpha$ such that $j_{i} \circ \phi_{i}: A_{i} \rightarrow A_{i} \times A_{i}$ is homotopic to $\Delta_{i}$ for $i=1,2$, where $\left(j_{1}, j_{2}\right): \alpha \vee \alpha \rightarrow \alpha \times \alpha$ is the inclusion and $\left(\Delta_{1}, \Delta_{2}\right): \alpha \rightarrow \alpha \times \alpha$ is the diagonal morphism. In this case, $\left(\phi_{1}, \phi_{2}\right)$ is called co-H-structure morphism of $\alpha$.

Example 2. If $\left(A_{1}, \mu_{1}\right)$ and $\left(A_{2}, \mu_{2}\right)$ are co-H-spaces and $\left(\mu_{1}, \mu_{2}\right): \alpha \rightarrow \alpha \vee \alpha$ is a morphism, then $\alpha: A_{1} \rightarrow A_{2}$ is a co-H-object. Thus if $f: C_{1} \rightarrow C_{2}$ is a map, then the suspension map $\Sigma f: \Sigma C_{1} \rightarrow \Sigma C_{2}$ is a co-H-object. Let $\phi_{i}: \Sigma C_{i} \rightarrow \Sigma C_{i} \vee \Sigma C_{i}$ be a co-H-structure given by

$$
\phi_{i}\langle c, t\rangle=\left\{\begin{array}{l}
(\langle c, 2 t\rangle, *) \text { if } 0 \leq t \leq 1 / 2 \\
(*,\langle c, 2 t-1\rangle) \text { if } 1 / 2 \leq t \leq 1
\end{array}\right.
$$

for $i=1,2$. Then we have the following commutative diagram:

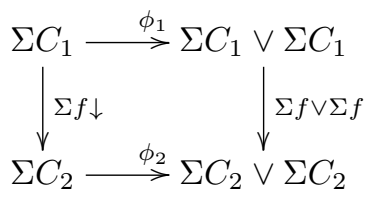

In fact,

$$
\phi_{2} \Sigma f\langle a, t\rangle=\phi_{2}(\langle f(a), t\rangle)= \begin{cases}(\langle f(a), 2 t\rangle, *) & \text { for } 0 \leq t \leq 1 / 2 \\ (*,\langle f(a), 2 t-1\rangle) & \text { for } 1 / 2 \leq t \leq 1\end{cases}
$$




$$
\begin{aligned}
(\Sigma f \vee \Sigma f) \phi_{1}\langle a, t\rangle & = \begin{cases}(\Sigma f \vee \Sigma f)(\langle a, 2 t\rangle, *) & \text { for } 0 \leq t \leq 1 / 2 \\
(\Sigma f \vee \Sigma f)(*,\langle a, 2 t-1\rangle) & \text { for } 1 / 2 \leq t \leq 1\end{cases} \\
& = \begin{cases}(\langle f(a), 2 t\rangle, *) & \text { for } 0 \leq t \leq 1 / 2 \\
(*,\langle f(a), 2 t-1\rangle) & \text { for } 1 / 2 \leq t \leq 1 .\end{cases}
\end{aligned}
$$

It is well-known fact if $\alpha$ is a suspension map, then $\Pi(\alpha, \beta)$ has a group structure for each object $\beta$.

Theorem 5.5. Let $\Sigma \alpha: \Sigma A_{1} \rightarrow \Sigma A_{2}$ be the suspension map of $\alpha: A_{1} \rightarrow A_{2}$, $\eta: X \rightarrow Y$ be a right invertible map with the inverse $\theta$ and $\left(h_{1}, h_{2}\right): \eta \rightarrow \Sigma \alpha$ be a morphism. Then $\mathcal{G}^{\left(h_{1}, h_{2}\right)}(\Sigma \alpha, \beta)$ is a subgroup of $\Pi(\Sigma \alpha, \beta)$ for each map $\beta: B_{1} \rightarrow B_{2}$.

Proof. By Lemma 4.1, it is sufficient to show that the group operation of $\Pi(\Sigma \alpha, \beta)$ is closed in $\mathcal{G}^{\left(h_{1}, h_{2}\right)}(\Sigma \alpha, \beta)$. Let $\left[f_{1}, f_{2}\right]$ and $\left[g_{1}, g_{2}\right]$ be elements in $\mathcal{G}^{\left(h_{1}, h_{2}\right)}(\Sigma \alpha, \beta)$. Then there are two $\left(h_{1}, h_{2}\right)$-affiliated morphisms $\left(H_{1}, H_{2}\right)$ : $\Sigma \alpha \times \eta \rightarrow \beta$ and $\left(G_{1}, G_{2}\right): \Sigma \alpha \times \eta \rightarrow \beta$ of $\left(f_{1}, f_{2}\right)$ and $\left(g_{1}, g_{2}\right)$, respectively. Consider the adjoint maps $\widetilde{H_{1}}, \widetilde{G_{1}}: A_{1} \rightarrow B_{1}^{X}$ of $H_{1}$ and $G_{1}$ respectively and $\widetilde{H_{2}}, \widetilde{G_{2}}: A_{2} \rightarrow B_{2}^{Y}$ of $H_{2}$ and $G_{2}$ respectively. Then we have the following commutative diagram:

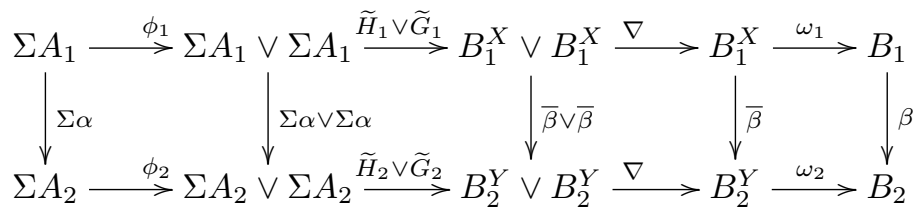

where $\left(\phi_{1}, \phi_{2}\right): \Sigma \alpha \rightarrow \Sigma \alpha \vee \Sigma \alpha$ is a co-H-structure morphism and $\bar{\beta}: B_{1}^{X} \rightarrow B_{2}^{Y}$ is given by $\bar{\beta}(g)=\beta g \theta$ for each $g \in B_{1}^{X}$. In order to show that the diagram is commutative, it is sufficient to check the commutativity of the second rectangle. Since $\beta\left(\widetilde{H_{1}}(a)(x)\right)=\beta\left(H_{1}(a, x)\right)=H_{2}(\Sigma \alpha(a), \eta(x))=\widetilde{H_{2}}(\Sigma \alpha(a))(\eta(x))$, we have

$$
\begin{aligned}
(\bar{\beta} \vee \bar{\beta})\left(\widetilde{H_{1}} \vee \widetilde{G_{1}}\right)(a, *) & =\left(\bar{\beta} \widetilde{H_{1}}(a), \bar{\beta} \widetilde{G_{1}}(*)\right) \\
& =\left(\beta \widetilde{H_{1}}(a) \theta, \bar{\beta}\left(h_{1}\right)\right) \\
& =\left(\widetilde{H_{2}} \Sigma \alpha(a) \eta \theta, h_{2}\right)
\end{aligned}
$$

$$
\left(\widetilde{H_{2}} \vee \widetilde{G_{2}}\right)(\Sigma \alpha \vee \Sigma \alpha)(a, *)=\left(\widetilde{H_{2}} \Sigma \alpha(a), \widetilde{G_{2}} \Sigma \alpha(*)\right)=\left(\widetilde{H_{2}} \Sigma \alpha(a), h_{2}\right)
$$

for each $(a, *) \in \Sigma A_{1} \vee \Sigma A_{1}$. Similarly, we can prove the equality for each

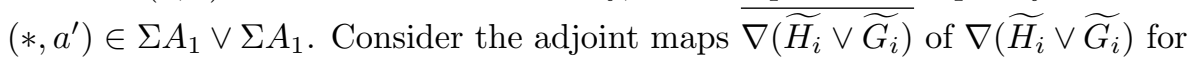


$i=1,2$. Then we have the following diagram:

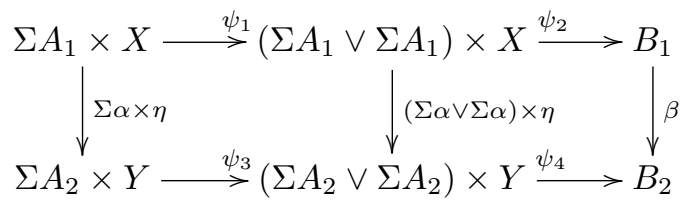

where $\psi_{1}=\phi_{1} \times 1_{X}, \psi_{2}=\overline{\nabla\left(\widetilde{H_{1}} \vee \widetilde{G_{1}}\right)}, \psi_{3}=\phi_{2} \times 1_{Y}$ and $\psi_{4}=\overline{\nabla\left(\widetilde{H_{2}} \vee \widetilde{G_{2}}\right)}$. Let us represent each element in $\Sigma A_{1}$ ( or $\Sigma A_{2}$ ) as $\langle a, t\rangle$. Then we have

$$
\begin{aligned}
& \left.\beta \overline{\nabla\left(\widetilde{H_{1}} \vee \widetilde{G_{1}}\right)}\left(\phi_{1} \times 1_{X}\right)(\langle a, t\rangle, x)=\beta \overline{\nabla\left(\widetilde{H_{1}} \vee \widetilde{G_{1}}\right.}\right)\left(\phi_{1}(\langle a, t\rangle), x\right) \\
& =\beta \nabla\left(\widetilde{H_{1}} \vee \widetilde{G_{1}}\right)\left(\phi_{1}(\langle a, t\rangle)\right)(x) \\
& =\beta \nabla\left(\widetilde{H_{1}} \vee \widetilde{G_{1}}\right)(\langle a, 2 t\rangle, *)(x) \\
& =\beta \widetilde{H_{1}}(\langle a, 2 t\rangle)(x) \\
& =\beta H_{1}(\langle a, 2 t\rangle, x) \\
& =H_{2}(\Sigma \alpha \times \eta)(\langle a, 2 t\rangle, x) \\
& =\widetilde{H_{2}}(\langle\alpha(a), 2 t\rangle)(\eta(x)) \\
& =\nabla\left(\widetilde{H_{2}} \vee \widetilde{G_{2}}\right)(\langle\alpha(a), 2 t\rangle, *)(\eta(x)) \\
& =\nabla\left(\widetilde{H_{2}} \vee \widetilde{G_{2}}\right)\left(\phi_{2}\langle\alpha(a), t\rangle\right)(\eta(x)) \\
& =\overline{\nabla\left(\widetilde{H_{2}} \vee \widetilde{G_{2}}\right)}\left(\phi_{2}\langle\alpha(a), t\rangle, \eta(x)\right) \\
& =\overline{\nabla\left(\widetilde{H_{2}} \vee \widetilde{G_{2}}\right)} \phi_{2}(\Sigma \alpha \times \eta)(\langle a, t\rangle, x)
\end{aligned}
$$

for $0 \leq t \leq 1 / 2$. By the same way we can prove the equality for the case $1 / 2 \leq t \leq 1$. Furthermore,

$$
\begin{aligned}
\left.\overline{\nabla\left(\widetilde{H_{1}} \vee \widetilde{G_{1}}\right.}\right)\left(\phi_{1} \times 1_{X}\right)(\langle a, t\rangle, *) & \left.=\overline{\nabla\left(\widetilde{H_{1}} \vee \widetilde{G_{1}}\right.}\right)\left(\phi_{1}(\langle a, t\rangle), *\right) \\
& =\nabla\left(\widetilde{H_{1}} \vee \widetilde{G_{1}}\right)\left(\phi_{1}(\langle a, t\rangle)\right)(*) \\
& =\omega_{1}\left(\nabla\left(\widetilde{H_{1}} \vee \widetilde{G_{1}}\right) \phi_{1}\right)(\langle a, t\rangle) \\
& =\omega_{1}\left(\widetilde{H_{1}}+\widetilde{G_{1}}\right)(\langle a, t\rangle),
\end{aligned}
$$

where ' + ' is the group operation in $\Pi\left(\Sigma A_{1}, B_{1}^{X}\right)$. Similarly,

$$
\overline{\nabla\left(\widetilde{H_{2}} \vee \widetilde{G_{2}}\right)}\left(\phi_{2} \times 1_{Y}\right)(\langle a, t\rangle, *)=\omega_{2}\left(\widetilde{H_{2}}+\widetilde{G_{2}}\right)(\langle a, t\rangle) \text {. }
$$

Since

$$
\begin{aligned}
\overline{\nabla\left(\widetilde{H_{1} \vee \widetilde{G_{1}}}\right)}\left(\phi_{1} \times 1_{X}\right)(*, x) & \left.=\overline{\nabla\left(\widetilde{H_{1}} \vee \widetilde{G_{1}}\right.}\right)\left(\phi_{1}(*), x\right) \\
& =\left(\nabla\left(\widetilde{H_{1}}(*), \widetilde{G_{1}}(*)\right)(x)=h_{1}(x)\right.
\end{aligned}
$$

and $\left.\overline{\nabla\left(\widetilde{H_{2}} \vee \widetilde{G_{2}}\right.}\right)\left(\phi_{2} \times 1_{Y}\right)(*, y)=h_{2}(y)$,

$$
\left[\omega_{1}\left(\widetilde{H_{1}}+\widetilde{G_{1}}\right), \omega_{2}\left(\widetilde{H_{2}}+\widetilde{G_{2}}\right)\right] \in \mathcal{G}^{\left(h_{1}, h_{2}\right)}(\Sigma \alpha, \beta) .
$$


But

$$
\begin{aligned}
\left(\omega_{1}, \omega_{2}\right)_{*}\left(\left[\widetilde{H_{1}}, \widetilde{H_{2}}\right]+\left[\widetilde{G_{1}}, \widetilde{G_{2}}\right]\right) & =\left[\omega_{1} \widetilde{H_{1}}, \omega_{2} \widetilde{H_{2}}\right]+\left[\omega_{1} \widetilde{G_{1}}, \omega_{2} \widetilde{G_{2}}\right] \\
& =\left[f_{1}, f_{2}\right]+\left[g_{1}, g_{2}\right] \\
& =\left[f_{1}+g_{1}, f_{2}+g_{2}\right] \in \mathcal{G}^{\left(h_{1}, h_{2}\right)}(\Sigma \alpha, \beta) .
\end{aligned}
$$

\section{References}

[1] D. H. Gottlieb, A certain subgroup of the fundamental group, Amer. J. Math. 87 (1965), $840-856$.

[2] _ Evaluation subgroups of homotopy groups, Amer. J. Math. 91 (1969), 729-756.

[3] _ Covering transformations and universal fibrations, Illinois J. Math. 13 (1969), $432-437$.

[4] P. J. Hilton, Homotopy Theory and Duality, Mimeographed Notes, Cornell Univ. Ithaca, NY, 1959.

[5] G. E. Lang, Evaluation subgroups of factor spaces, Pacific J. Math. 42 (1972), 701-709.

[6] K. Y. Lee and M. H. Woo, The G-sequence and the $\omega$-homology of a CW-pair, Topology Appl. 52 (1993), no. 3, 221-236.

[7] _ Cyclic morphisms in the category of pairs and generalized G-sequences, J. Math. Kyoto Univ. 38 (1998), no. 2, 271-285.

[8] K. Y. Lee, M. H. Woo, and X. Zhao, Certain generalizations of G-sequences and their exactness, Bull. Korean Math. Soc. 45 (2008), no. 1, 119-131.

[9] K. L. Lim, On cyclic maps, J. Austral. Math. Soc. Ser. A 32 (1982), no. 3, 349-357.

[10] N. Oda, The homotopy set of the axes of pairings, Canad. J. Math. 42 (1990), no. 5, 856-868.

[11] J. Pan, X. Shen, and M. Woo, The G-sequence of a map and its exactness, J. Korean Math. Soc. 35 (1998), no. 2, 281-294.

[12] J. Siegel, G-spaces, W-spaces and H-spaces, Pacific J. Math. 31 (1969), 209-214.

[13] K. Varadarajian, Generalised Gottlieb groups, J. Indian Math. Soc. (N.S.) 33 (1969), $141-164$.

[14] M. Woo and J. Kim, Certain subgroups of homotopy groups, J. Korean Math. Soc. 21 (1984), no. 2, 109-120.

[15] M. H. Woo and K. Y. Lee, On the relative evaluation subgroups of a CW-pair, J. Korean Math. Soc. 25 (1988), no. 1, 149-160.

JIYEAN KIM

Department of Mathematics

KOREA UnIVERSITY

SeOul 136-701, Korea

E-mail address: eskim@korea.ac.kr

Kee Young Lee

DePARTMENT OF InFORMATION AND MATHEMATICS

Korea UnIVERsity

Chochieon 339-700, Korea

E-mail address: keyolee@korea.ac.kr 